\title{
Hybrid Capture 2 High-Risk HPV DNA Test
}

National Cancer Institute

\section{Source}

National Cancer Institute. Hybrid Capture 2 High-Risk HPV DNA Test. NCI Thesaurus.

Code $C 122035$.

A proprietary in vitro nucleic acid hybridization-based assay kit that can demonstrate the absence or presence of DNA from 13 types of human papillomavirus (HPV) in cervical specimens. 